\title{
CARACTERIZAÇÃO DA MUCILAGEM DE JARACATIÁ (CARICA QUERCIFOLIA (A. ST.-HIL.) HIERON) LIOFILIZADA PARA USO EM PROCESSOS DE SEPARAÇÃO
}

\author{
H. M. HEIDEMANN ${ }^{1}$, C. FACCIO ${ }^{1}$, M. G. N. QUADRI ${ }^{1}$, U. SIMÃO ${ }^{1}$ e S. R. ZOLDAN ${ }^{2}$ \\ ${ }^{1}$ Universidade Federal de Santa Catarina, Departamento de Engenharia Química e Engenharia \\ de Alimentos \\ ${ }^{2}$ Empresa de Pesquisa Agropecuária e Extensão Rural de Santa Catarina - EPAGRI, Campos \\ Novos/SC \\ E-mail para contato: mara@enq.ufsc.br
}

\begin{abstract}
RESUMO - O jaracatiá (Carica quercifolia (A. St.-Hil.) Hieron) pertence à família Caricaceae e ao gênero Vasconcellea, o qual possui 21 espécies. O foco do presente estudo é a caracterização da sua mucilagem, pois poucos são os dados encontrados e a partir destes vislumbrar possíveis aplicações, corroborando com a preservação da espécie e o aproveitamento de novas fontes vegetais. Foi determinada a composição química, incluindo análises dos grupos funcionais, da estrutura do sólido e o comportamento térmico. A mucilagem de jaracatiá liofilizada apresentou $2,9 \%$ de umidade, $9,8 \%$ de cinzas, $15,8 \%$ de fibra alimentar, $36,4 \%$ de carboidratos totais, $16 \%$ de açúcares redutores, $3,5 \%$ de lipídeos e $15,6 \%$ de proteínas. A atividade antioxidante foi de 26,6 \% DPPH sequestrado e o teor de fenólicos de 173,2 mg/L EAG. Os principais grupos funcionais observados no FTIR foram O$\mathrm{H}, \mathrm{C}-\mathrm{H}, \mathrm{C}=\mathrm{O}$ e C-O. Em relação à TGA/DTG a mucilagem demonstrou maior perda de massa, cerca de $50 \%$, à temperatura média de $300{ }^{\circ} \mathrm{C}$. A curva de estabilidade térmica por DSC mostrou que acima de $300{ }^{\circ} \mathrm{C}$ ocorre a degradação do material. A mucilagem de jaracatiá liofilizada apresentou características de um material semicristalino.
\end{abstract}

\section{INTRODUÇÃO}

Os gêneros Carica (uma espécie), Horovitzia (uma espécie), Jacaratia (sete espécies), Jarilla (três espécies) e Vasconcellea (21 espécies) são originários do continente americano, enquanto o gênero Cylicomorpha (duas espécies) pertence ao continente africano (VAN DROOGENBROECK et al., 2004), no entanto todos pertencem à família Caricaceae. Outros nomes utilizado para identificar a Carica quercifolia (A. St.-Hil.) Hieron são Vasconcellea quercifolia A. St.-Hil. e Carica quercifolia Solms-Laub. (COLOMBO et al., 1989), e os nomes populares pelos quais é conhecida são jaracatiá, jacaratiá, mamute, mamão-do-mato, mamoeiro-do-mato, mamãozinho-do-mato, mamãozinho, mamoeirinho e mamoeiro-bravo (KINUPP, 2007).

Essa espécie, no entanto, está em risco de extinção em virtude, provavelmente, do fato de a indústria de doces caseiros usar partes do seu caule em substituição à polpa do fruto de coco. Hoje, o jaracatiá é encontrado nas fazendas tradicionais, onde a vegetação nativa foi preservada, 
ou nos viveiros de alguns centros de pesquisa, sendo importantes estudos para viabilizar sua propagação e a conservação de material genético (FREITAS et al., 2011).

A mucilagem é constituinte natural e não é indicação de alterações da planta. Ocorre geralmente nas sementes, nas quais parece ter a função de reter água para facilitar a germinação, mas pode ocorrer também em outras partes do vegetal (SIMÕES et al., 2007). É uma fração da composição polissacarídica das plantas, capaz de se tornar viscosa na presença de água (CÁRDENAS et al., 1998). Ocorre geralmente nas sementes, nas quais parece ter a função de reter água para facilitar a germinação, mas pode ocorrer também em outras partes do vegetal (SIMÕES et al., 2007).

A caracterização da mucilagem liofilizada é o objetivo deste estudo, a qual se faz necessária, visto que os dados referentes à espécie Carica quercifolia (A. St.-Hil.) Hieron são escassos na literatura. A investigação destas características irá servir de base para vislumbrar possíveis aplicações, com o intuito tanto de preservação ambiental da espécie, como de aproveitamento de novas fontes vegetais (biodiversidade brasileira).

\section{MATERIAIS E MÉTODOS}

\subsection{Materiais}

Os jaracatiás (Carica quercifolia (A. St.-Hil.) Hieron) foram obtidos através da EPAGRI - Campos Novos/SC e enviados ao LASIPO/ENQ/UFSC para obtenção da mucilagem, os quais permaneceram congelados até o momento da extração. Uma exsicata da espécie foi depositada no Herbário do Instituto de Biociências (Herbário ICN) da Universidade Federal do Rio Grande do Sul sob o número de tombo ICN 173708. Todos os experimentos foram realizados em duplicata.

\subsection{Métodos}

Extração: A mucilagem bruta (proporção polpa com as sementes/água) de 1:1,25 foi extraída do jaracatiá descascado e triturado brevemente em liquidificador, após foi realizada a filtração em malha de poliéster com auxílio de vácuo. A mucilagem bruta foi liofilizada em liofilizador marca Liotop, modelo L101, Brasil; para posterior realização das análises. A liofilização foi realizada no Laboratório de Propriedades Físicas de Alimentos do Departamento de Engenharia de Alimentos da Universidade Federal de Santa Catarina.

Rendimento: Os frutos foram pesados, descascados e triturados. A mucilagem foi extraída da polpa com as sementes, e submetida ao processo de liofilização. Em seguida, o material foi novamente pesado a fim de verificar o rendimento total da mucilagem liofilizada.

Composição química: Umidade: secagem direta em estufa $105^{\circ} \mathrm{C}$ (IAL, 2008). Lipídios: método enzimático-colorimétrico Analisa, comercializado por Gold Analisa Diagnóstica Ltda. Proteína total: baseia-se na determinação de nitrogênio pelo processo de digestão Kjeldahl (GALVANI; GAERTNER, 2006) com algumas modificações. Cinzas: incineração a $550{ }^{\circ} \mathrm{C}$ (IAL, 2008). Carboidratos Totais: fenol sulfúrico (DUBOIS et al., 1956) e açúcares redutores: ácido dinitrosalicílico (DNS) (MILLER, 1959). Fibra alimentar: método enzimático- 
gravimétrico (AOAC 991.43, 1995). Compostos fenólicos (SINGLETON; ROSSI, 1965). Atividade antioxidante (BRAND-WILLIANS et al., 1995), com algumas modificações.

Espectroscopia no infravermelho com transformada de Fourier (FTIR): As amostras foram analisadas por espectroscopia na região do infravermelho com transformada de Fourier (FTIR), em um espectrofotômetro FTIR Shimadzu modelo IRP Prestige-21 com detector DLATGS, com faixa espectral de 400 a $4000 \mathrm{~cm}^{-1}$ e resolução de $2 \mathrm{~cm}^{-1}$, por transmissão em pastilhas de $\mathrm{KBr}$ de $7 \mathrm{~mm}$ de diâmetro (200-300 mg). A análise foi realizada no Laboratório de Controle de Processos do Departamento de Engenharia Química da Universidade Federal de Santa Catarina.

Análise térmica (TGA, DTG e DSC): Para a realização da análise de calorimetria diferencial de varredura (DSC) foram utilizadas cerca de 5 - $10 \mathrm{mg}$ de amostra, as quais foram colocadas em cadinho de platina e analisadas em equipamento Netzsch STA modelo 449 F3 Jupiter com faixa de temperatura de $25{ }^{\circ} \mathrm{C}$ a $700{ }^{\circ} \mathrm{C}$, taxa de aquecimento de $10{ }^{\circ} \mathrm{C} \cdot \mathrm{min}^{-1} \mathrm{e}$ vazão de nitrogênio de $20 \mathrm{~mL} \cdot \mathrm{min}^{-1}$. Para a análise termogravimétrica (TGA) e a derivada (DTG) foram utilizadas as mesmas condições de operação, visto que o equipamento é acoplado. A análise foi realizada no Laboratório de Controle de Processos do Departamento de Engenharia Química da Universidade Federal de Santa Catarina.

Difratometria de raio X (DRX): Foi realizada em difratômetro marca PANanalytical modelo X'Pert PRO MPD, com variação angular de $4^{\circ}$ a $80^{\circ}(2 \theta)$, operado em $30,0 \mathrm{kV}, 30,0$ $\mathrm{mA}$ e passo de $0,33^{\circ}$ a cada 20 segundos, de acordo com metodologia utilizada por Branco (2011), com algumas modificações. A análise foi realizada no Laboratório Multiusuário de Difração de Raio X do Departamento de Física da Universidade Federal de Santa Catarina.

\section{RESULTADOS E DISCUSSÃO}

\subsection{Rendimento}

Para avaliação do rendimento foram utilizadas, em cada batelada, $300 \mathrm{~g}$ de jaracatiás (Carica quercifolia (A. St.-Hil.) Hieron). Depois de descascados, o peso total da polpa com as sementes foi de aproximadamente $200 \mathrm{~g}$, apresentando, assim, um rendimento médio de $66,67 \%$ de polpa/sementes e 33,33\% de casca. Após a extração da mucilagem bruta e liofilização o rendimento foi em torno de $5 \%$.

\subsection{Composição proximal}

Na Tabela 1 são mostrados os resultados obtidos da composição proximal da mucilagem de jaracatiá (Carica quercifolia (A. St.-Hil.) Hieron) liofilizada, e de forma comparativa valores encontrados por outros autores para outras mucilagens, pois não foi encontrado nenhum estudo com a avaliação da composição proximal da mucilagem em estudo. 
Tabela 1 - Composição proximal da mucilagem de jaracatiá liofilizada.

\begin{tabular}{|c|c|c|c|}
\hline \multirow{2}{*}{ Componente } & \multicolumn{3}{|c|}{ Mucilagem } \\
\cline { 2 - 4 } & Jaracatiá* & $\begin{array}{c}\text { Inhame (TAVARES } \\
\text { et al., 2011) }\end{array}$ & $\begin{array}{c}\text { Chia (CAPITANI et } \\
\text { al., 2013) }\end{array}$ \\
\hline Umidade (\%) & $2,91 \pm 0,49$ & 8,68 & $11,5 \pm 3,0$ \\
\hline Cinzas (\%) & $9,85 \pm 0,03$ & 5,33 & $8,4 \pm 1,0$ \\
\hline Fibra Alimentar (\%) & $5,35 \pm 0,11$ & 10,45 & $13,5 \pm 6,0$ \\
\hline Carboidratos (\%) & $36,43 \pm 1,96$ & 65,18 & $63,7 \pm 5,0$ \\
\hline Açúcares redutores (\%) & $16,01 \pm 1,52$ & n.a. & n.a. \\
\hline Proteína (\%) & $15,57 \pm 1,83$ & 9,66 & $3,1 \pm \pm 3,0$ \\
\hline Lipídios (\%) & $3,55 \pm 0,50$ & 0,7 & \\
\hline
\end{tabular}

*base seca; n.a. - não analisado

A concentração obtida para compostos fenólicos da mucilagem de jaracatiá (Carica quercifolia (A. St.-Hil.) Hieron) liofilizada foi de $173 \pm 3,97 \mathrm{mg} . \mathrm{L}^{-1}$ EAG (equivalente ácido gálico) e a atividade antioxidante foi de 26,66 $\pm 1,3 \%$ DPPH sequestrado. Como podemos observar, em relação aos compostos fenólicos, a mucilagem de jaracatiá liofilizada apresentou

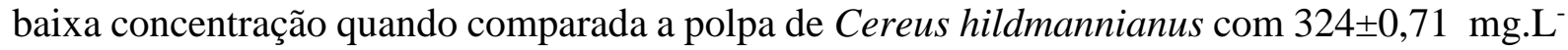
${ }^{1}$ EAG (PILETTI, 2011), ao yacon com 635 $\pm 20,82 \mathrm{mg} . \mathrm{L}^{-1}$ EAG (VANDRESEN, 2011) e a da acerola com 3366,55 $\pm 67,33 \mathrm{mg} . \mathrm{L}^{-1} \mathrm{EC}$ (equivalente catequina) (MELO et al., 2008).

A avaliação da atividade antioxidante em extratos de frutas utilizou a classificação adotada por Melo et al. (2008): acima de 70\% de sequestro como forte ação antioxidante, entre 50 e $70 \%$ moderada e abaixo de $50 \%$ como fraca. Seguindo esta classificação a mucilagem de jaracatiá liofilizada mostrou fraca ação antioxidante, o que pode ser atribuído ao processo de liofilização e tempo de armazenamento, já que os antioxidantes são muito lábeis e fotossensíveis (TAVARES et al., 2011), principalmente as reações de oxidação (ROBARDS et al., 1999).

\subsection{Espectroscopia na região do infravermelho com transformada de Fourier (FTIR)}

O espectro na região do infravermelho obtido da mucilagem de jaracatiá liofilizada é mostrado na Figura 1.

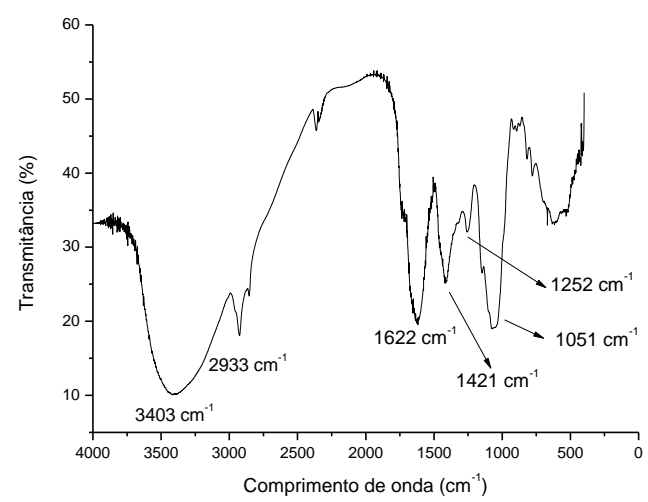

Figura 1 - Espectro de FTIR da mucilagem de jaracatiá liofilizada. 
O espectro da mucilagem de jaracatiá (Carica quercifolia (A. St.-Hil.) Hieron) liofilizada apresentou uma banda forte e larga em $3403 \mathrm{~cm}^{-1}$, que pode ser atribuída ao estiramento vibracional do grupo O-H de água e álcoois (SILVERSTEIN et al., 2006; PAVIA et al, 2008; YUEN et al., 2009). Também são encontradas duas bandas de intensidade baixa na faixa de absorção a 2900-2800 $\mathrm{cm}^{-1}$, que podem ser atribuídas às vibrações simétricas e assimétricas do grupamento C-H (SILVERSTEIN et al., 2006; TAVARES et al., 2011). A banda de média intensidade em $1622 \mathrm{~cm}^{-1}$ pode ser do grupo $\mathrm{C}=\mathrm{O}$ (PAVIA et al., 2008; TAVARES et al., 2011). A banda em $1421 \mathrm{~cm}^{-1}$ pode ser atribuída às deformações simétricas dos grupos $\mathrm{C}-\mathrm{H} \mathrm{e}$ C-OH (WANG; SOMASUNDARAN, 2006). A banda em $1252 \mathrm{~cm}^{-1}$ corresponde ao estiramento C-O em polissacarídeos (SILVERSTEIN et al., 2006; CAI et al., 2008). A banda em $1051 \mathrm{~cm}^{-1}$ pode ser relacionada à deformações simétricas e assimétricas do grupo $\mathrm{C}-\mathrm{OH}$. (TAVARES et al., 2011).

\subsection{Análise térmica (DSC, TGA e DTG)}

As curvas da DSC, TGA e DTG obtidas da mucilagem de jaracatiá liofilizada são mostradas na Figura 2.

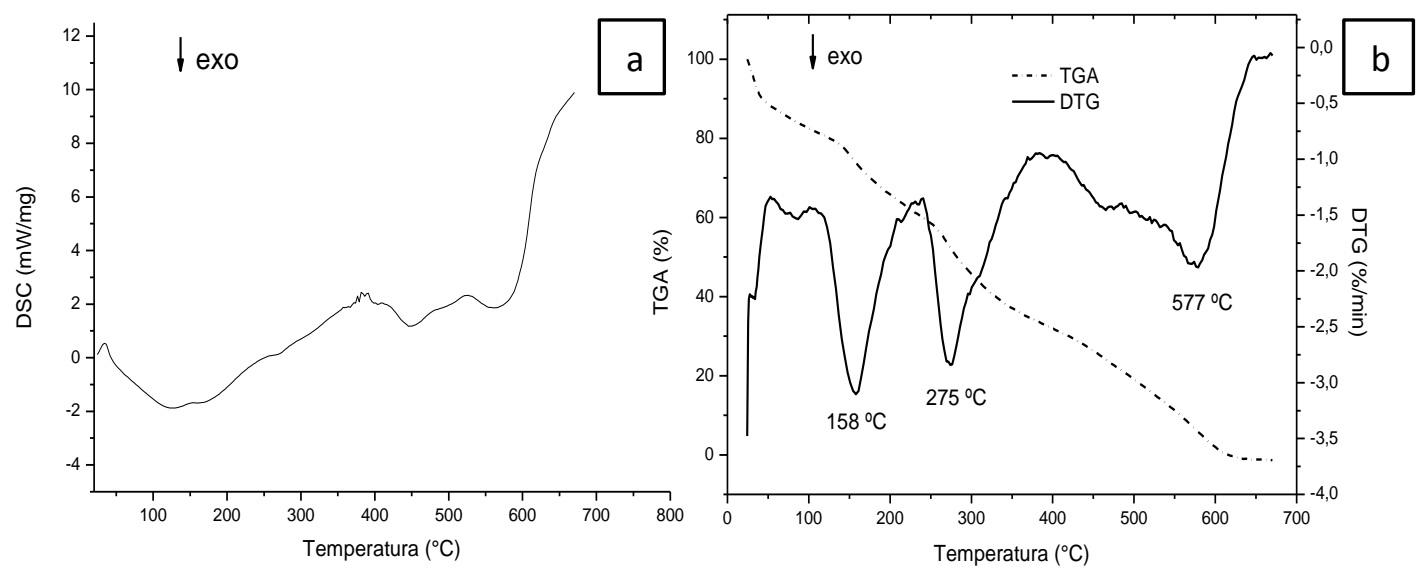

Figura 2 - Curvas de DSC (a) e TGA/DTG (b) da mucilagem de jaracatiá (Carica quercifolia (A. St.-Hil.) Hieron) liofilizada.

A curva de estabilidade térmica (Figura 2 (a)) por DSC da mucilagem de jaracatiá liofilizada apresenta três transições térmicas, uma endotérmica na faixa de $0-100{ }^{\circ} \mathrm{C}$, associado à evaporação da água. Os eventos exotérmicos acima de $300^{\circ} \mathrm{C}$ são atribuídos à degradação do material (MACEDO, 2006; VENDRUSCOLO et al., 2009; BRANCO, 2011).

De acordo com a Figura 2 (b) podemos observar que a primeira perda de massa ocorre entre $0-150{ }^{\circ} \mathrm{C}$, a qual pode ser atribuída à evaporação de água contida no polímero (KITTUR et al., 2002; TAVARES et al., 2011; SUN et al., 2011), em concordância com o resultado obtido pela DSC. As perdas de massa subsequentes de $30 \%$ e $50 \%$ podem estar atribuídas à decomposição dos polissacarídeos (ZOHURIAAN; SHOKROLAHI, 2004; TAVARES et al., 2011). 


\subsection{Difratometria de raio $\mathrm{X}(\mathrm{DRX})$}

O difratograma obtido da mucilagem de jaracatiá liofilizada é mostrado na Figura 3.

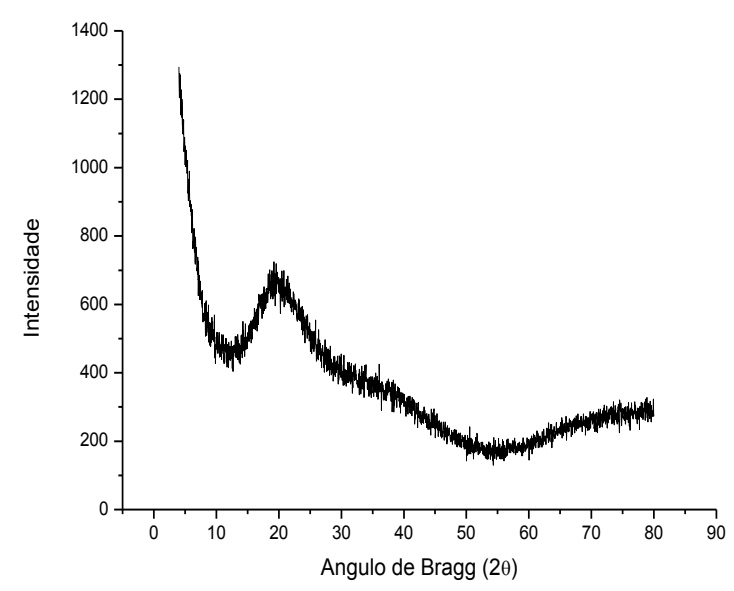

Figura 3 - Difratograma da mucilagem de jaracatiá liofilizada.

Como podemos visualizar na Figura 3, a mucilagem de jaracatiá (Carica quercifolia (A. St.-Hil.) Hieron) liofilizada apresenta características de um material semicristalino, o que pode ser observado na faixa de $10^{\circ}<2 \theta<80^{\circ}$, mesmo comportamento foi observado por Mishra $e t$ al. (2006) para a mucilagem de feno-grego e Branco (2011) para a mucilagem do cladódio de Cereus hildmaniannus K. Schum.

\section{CONCLUSÃO}

Nas condições experimentais em que foi realizado o presente trabalho, os resultados obtidos permitiram concluir que: o rendimento médio da mucilagem de jaracatiá (Carica quercifolia (A. St.-Hil.) Hieron) liofilizada foi de 5\%; a mucilagem de jaracatiá liofilizada apresenta teores consideráveis de proteína bruta, fibra alimentar, açúcares redutores, cinzas e carboidratos totais; e baixo teor de gordura; os compostos fenólicos apresentaram baixa concentração e a atividade antioxidante foi fraca; os principais grupos funcionais encontrados na mucilagem de jaracatiá liofilizada foram $\mathrm{OH}, \mathrm{C}-\mathrm{H}, \mathrm{C}=\mathrm{O}$ e $\mathrm{C}-\mathrm{O}$; o comportamento da mucilagem de jaracatiá liofilizada na análise termogravimétrica, demonstra maior perda de massa cerca de $50 \%$ à temperatura média de $300^{\circ} \mathrm{C}$, o que inviabiliza o seu uso acima dessa temperatura e a curva de estabilidade térmica por DSC mostrou que acima de $300{ }^{\circ} \mathrm{C}$ ocorre a degradação do material; a mucilagem de jaracatiá liofilizada apresentou características de um material semicristalino.

\section{REFERÊNCIAS BIBLIOGRÁFICAS}

AOAC - Association of Official Agricultural Chemists. Official methods of the Association of the Agricultural Chemists. 16. ed. Washington, DC, v. 2, Fibra alimentar - $\mathrm{n}^{\circ}$ 991.43, 1995. 
BRANCO, N. B. C. Mucilagem do cladódio de Cereus hildmaniannus K. Schum: caracterização física, química e reológica. Dissertação (Mestrado em Engenharia Química) Departamento de Engenharia Química e de Alimentos, Universidade Federal de Santa Catarina, Florianópolis/SC, 2011.

BRAND-WILLIAMS, W.; CUVELIER, M.E.; BERSET, C. Use of free radical method to evaluate antioxidant activity. Lebensm. Wiss. Technol., v.28, p.25-30, 1995.

CAI, W.; GU, X.; TANG, J. Extraction, purification, and characterization of the polysaccharides from Opuntia milpa alta. Carbohydr. Polym., v. 71, p. 403-410, 2008.

CAPITANI, M. I.; IXTAINA, V. Y.; NOLASCO, S. M.; TOMÁS, M. C. Microstructure, chemical composition and mucilage exudation of chia (Salvia hispanica L.) nutlets from Argentina. J. Sci. Food Agric., v. 93, p. 3856-3862, 2013.

CÁRDENAS, A.; ARGUELLES, W. M.; GOYCOOLEA, F. On the posible rol of Opuntia ficus-indica Mucilage in Lime Mortar Perfomance in the Protection of Historical Buildings. $J$. Profess. Assoc. Cactus Developm., v. 3, 1998.

COLOMBO, P.; MELATI, M. R.; SCIALABBA, A.; TRAPANI, S.; SORTINO, M. The ecomorphology of Carica quercifolia Solms-Laub. in a Mediterranean climate. Agric. Ecosyst. Environ., v. 27, p. 397-409, 1989.

DUBOIS, M.; GILlES, K. A.; HAMILTON, J. K.; REBERS, P. A.; SMITH, F. Colorimetric method for determination of sugars and related substances. Anal. Chem., v. 28, p. 350-428, 1956.

FREITAS, S. J.; BARROSO, D. G.; SILVA, R. F. DA; MARTINS, V. H. C. R.; FREITAS, M. D. S.; FERREIRA, P. R. Métodos de remoção da sarcotesta na germinação de sementes de Jaracatiá. Rev. Árvore, v. 35, p. 91-96, 2011.

GALVANI, F.; GAERTNER, E. Adequação da metodologia Kjeldahl para determinação de nitrogênio total e proteína bruta. EMBRAPA Pantanal - Circular Técnica 63, 2006.

IAL - Instituto Adolfo Lutz. Normas Analíticas do Instituto Adolfo Lutz. Métodos físicoquímicos para análise de alimentos, $4^{\mathrm{a}}$ ed., $1^{\mathrm{a}}$ ed. online, São Paulo, 2008.

KINUPP, V. F. Plantas alimentícias n-convencionais da região metropolitana de Porto Alegre, $R S$. Tese (Doutorado em Fitotecnia) - Faculdade de Agronomia, Universidade Federal do Rio Grande do Sul, Porto Alegre/RS, 2007.

KITTUR, F. S.; HARISH PRASHANTH, K. V.; UDAYA SANKAR, K.; THARANATHAN, R. N. Characterization of chitin, chitosan and their carboxymethyl derivatives by differential scanning calorimetry. Carbohydr. Polym., v. 49, p. 185-193, 2002.

MACÊDO, A. A. M. Filmes de colágeno-polissacarídeo sulfatado como matrizes com propriedades antitrombogênicas: preparação e caracterização. Dissertação (Mestrado em Bioquímica) - Departamento de Bioquímica e Biologia Molecular, Universidade Federal do Ceará, Fortaleza/CE, 2006.

MELO, E. A.; MACIEL, M. I. S.; LIMA, V. L. A. G.; NASCIMENTO, R. J. Capacidade antioxidante de frutas. Rev. Bras. Ciênc. Farm., v. 44, p. 193-201, 2008.

MILLER, G. L. Use of dinitrosalicylic acid reagent for determination of reducing sugar. Anal. Chem., v. 31, p. 426-428, 1959. 
MISHRA, A.; YADAV, A.; PAL, S.; SINGH, A. Biodegradable graft copolymers of fenugreek mucilage and polyacrylamide: A renewable reservoir to biomaterials. Carbohydr. Polym., v. 65, p. 58-63, 2006.

PAVIA, D. L.; KRIZ, G. S.; LAMPMAN, G. M. Introduction to Spectroscopy. Fourth Edition. Brooks Cole, 2008.

PILETTI, R. Extração da mucilagem da tuna (Cereus hildmaniannus K. Schum) para aproveitamento industrial. Tese (Doutorado em Engenharia Química) - Universidade Federal de Santa Catarina, Florianópolis/SC, 2011.

ROBARDS, K.; PRENZLERA, P. D.; TUCKERB, G.; SWATSITANGB, P.; WILLIAM GLOVER, W. Phenolic compounds and their role in oxidative processes in fruits. Food Chem., v. 66, p. 401-436, 1999.

SIMÕES, C. M. O.; SCHENKEL, E. P.; GOSMANN, G.; MELLO, J. C. P.; MENTZ, L. A.; PETROVICK, P. R. Farmacognosia: da planta ao medicamento. Porto Alegre: UFRGS Editora, Florianópolis: Editora da UFSC, 2007, 1104 p.

SILVERSTEIN, R. M.; WEBSTER, F. X.; KIEMLE, D. J. Identificação espectrofotométrica de compostos orgânicos. $7^{\mathrm{a}}$ edição, Rio de Janeiro: LTC, 2006.

SINGLETON, V. L.; ROSSI, J. A. Colorimetry of total phenolics with phosphomolybdicphosphotungstic acid reagents. Am. J. Enol. Viticult., v. 20, p.144-158, 1965.

SUN, Y. C.; WEN, J. L.; XU, F.; SUN, R. C. Structural and thermal characterization of hemicelluloses isolated by organic solvents and alkaline solutions from Tamarix austromongolica. Bioresour. Technol., v. 102, p. 5947-5951, 2011.

TAVARES, S. A.; PEREIRA, J.; GUERREIRO, M. C.; PIMENTA, C. J.; PEREIRA, L.; MISSAGIA, S. V. Caracterização físico-química da mucilagem de inhame liofilizada. Ciênc. Agrotec., v. 35, p. 973 -979, 2011.

VAN DROOGENBROECK, B.; KYNDT, T.; MAERTENS, I.; ROMEIJN-PEETERS, E.; SCHELDEMAN, X.; ROMERO-MOTOCHI, J.; VAN DAMME, P.; GOETGHEBEUR, P.; GHEYSEN, G. Phylogenetic analysis of the highland papayas (Vasconcellea) and allied genera (Caricaceae) using PCR-RFLP. Theor. Appl. Genet., v. 108, p. 1473-1486, 2004.

VANDRESEN, S. Purificação parcial de inulina obtida a partir de yacon e recuperação de compostos antioxidantes por processos de sorção. Tese (Doutorado em Engenharia Química) - Universidade Federal de Santa Catarina, Florianópolis/SC, 2011.

WANG, J.; SOMASUNDARAN, P. Mechanisms of ethyl(hydroxyethyl) cellulose-solid interaction: Influence of hydrophobic modification. J. Colloid Interface Sci., v. 293, p. 322$332,2006$.

YUEN, S.-N.; CHOI, S.-M.; PHILLIPS, D. L.; MA, C.-Y. Raman and FTIR spectroscopy study of carboxymethylated non-starch polysaccharides. Food Chem., v. 114, p. 1091-1098, 2009.

ZOHURIAAN, M. J.; SHOKROLAHI, F. Thermal studies on natural and modified gums. Polym. Test., v. 23, p. 575-579, 2004. 